\title{
Don't blame the economists. It is an inverse problem!
}

\author{
Jaime Gomez-Ramirez
}

Received: 26 April 2013 / Accepted: 9 July 2013 /Published online: 4 August 2013

(C) The Author(s) 2013. This article is published with open access at Springerlink.com

\begin{abstract}
The seriousness of the current crisis urgently demands new economic thinking that breaks the austerity vs. deficit spending circle in economic policy. The core tenet of the paper is that the most important problems that natural and social science are facing today are inverse problems, and that a new approach that goes beyond optimization is necessary. The approach presented here is radical in the sense that it identifies the roots in key assumptions in economic theory such as optimal behavior and stability to provide an inverse thinking perspective to economic modeling, of use in economic and financial stability policy. The inverse problem provides a truly multidisciplinary platform where related problems from different disciplines can be studied under a common approach with comparable results.
\end{abstract}

Keywords Inverse problem - Complexity economics · Bio-inspired homeostasis $\cdot$ Subjective expected utility

\section{Introduction}

The relationship between economic aggregates such as the unemployment rate or inflation, based on direct measurement of quantities is an inverse problem which is not well posed. The inverse problem is to infer the value of parameters of interest based on the direct measurement of observables. This form of inference is ill-posed in the sense that solutions to the problem may not exist, be multiple or be instable, that is, small errors in the measurements lead to large differences in the solution. In order to be able to build on this fundamental but poorly understood insight we need to critically explore pillars of orthodox economic theory like the rational expectation

J. Gomez-Ramirez $(\bowtie)$

Universidad Politécnica de Madrid, José Gutiérrez Abascal, 2,

Madrid 28006, Spain

e-mail: jd.gomez@upm.es hypothesis, market efficiency and stable equilibrium, providing new avenues and hands-on alternatives for more realistic and resilient economic models. More specifically, we claim that the new generation of economists must be aware of the necessity to deal with the next three points:

1. Develop a new theoretical framework based on the inverse problem theory that will work as a scaffold, where key topics like stability, causality or predictability in economic models are addressed.

2. Establish a multidisciplinary program to gather and analyze data relative to cognitive and ecological features of interest for economic system modeling.

3. Develop new modeling techniques; mathematical and computational, including complexity theory tools e.g., network analysis to further our understanding of the mechanisms underlying robustness

\section{Four problems in classical economic modeling}

Economies are complex man-made systems where organisms and markets interact according to motivations and principles not entirely understood yet. The increasing dissatisfaction with the postulates of traditional economics i.e. perfectly rational agents interacting through efficient and stable markets, has created new incentives for different approaches in economics. For example, behavioral economics [1] builds on cognitive and emotional models of agents, neuroeconomics [2] addresses the neurobiological basis of valuation of choices, and evolutionary economics [3] studies economies as a complex evolutionary system, composed of agents that adapt to endogenous patterns out of equilibrium regions. Social science, and in particular economics, is undergoing a decisive historical moment. A new approach in economic theory able to palliate the dissatisfaction with core tenets in classical economics is sorely needed. In what follows we briefly describe four 
problems - agent-economy separation, causality, equilibrium and perfectly rational maximizers- that need to be specifically addressed.

\section{Agent-economy separation}

Orthodox economic thinking draws a neat line between agents and the economy they perform. To that effect microeconomics and macroeconomics are publicized as the suitable disciplines to address the issues related to the former and the last respectively. Samuelson changed forever the study of economics, which became strongly mathematical by borrowing methods and insights from statistical physics. University departments of economics are an institutionalized embodiment of his vision. However, the idiosyncrasy of economic systems composed of participants endowed with cognitive and emotional characteristics clearly calls into question the analogy between physics and economics. Economic agents are not merely reactive but have an anticipatory nature. Realistic economic systems try to predict the outcomes of their actions and of those of other agents, and in doing so they modify the structure itself that they are trying to cope with or outperform, as in financial markets. The forward-looking capacity of biological and social systems is an additional layer of complexity that statistical mechanics applications lack.

\section{Causality}

Econometric models have mainly focused on models of exchange and allocation, neglecting critical aspects in economies' dynamics like production or credit money. This apparent lax approach has its root in modeling economic systems "as if" [4] having an equivalent ontological status with the rest of material systems that form the "book of nature that is written in the language of mathematics", as Galileo poetically stated. However, the "unreasonable effectiveness of mathematics in physics" relies upon the existence of invariance principles, observed in quantities that remain constant regardless the system's motion [5]. Economies are not conservative systems, assets are created ex novo, as in the process of money creation through credit; and disappear, enacted by the innovative entry of new technologies. This is the disruptive force that sustains economic growth or Schumpeter's "creative destruction" [6]. Thus, variance principles and general laws in economics are more a desideratum than a factum. The Lucas critique [7] is still valid, even though the approaches that followed like dynamic stochastic general equilibrium (DGSE) forgo realism for mathematical virtuosity, macroeconometric models are not invariant to economic policy, or simply put, a policy experiment may modify the structural parameters that it is trying to predict. The causal thinking in economics, which mainly relies on "the genetic-causal tradition" [8] must be explored with fresh insight.

Equilibrium

In Newtonian physics and classical thermodynamics the laws that govern the behavior of single particles suffice for the study of assemblies of particles, but this "methodological bonus" comes with a cost, they are theories of equilibrium, and therefore do not apply to far-from-equilibrium systems. Equilibrium is a terminal state that is reached when all process have stopped. Biological and social systems are open systems that transition between different regimes, so nonstationarity and volatility have to be taken into account. The participants in economic systems are heterogeneous. They are equipped with different informational processing capabilities, different systems of belief and ways to internalize the world. Thus, their interactions may produce novel dynamics and patterns whose understanding lie outside the basic assumptions necessary to establish an equilibrium, such as perfect competition or perfect knowledge of all the participants. Participants in an economy cannot be merely averaged out as particles in a gas, as they try to adapt to complex, highly coupled and self-referential eco-systems. One unique stable equilibrium cannot be taken for granted, instead multiple equilibria and qualitative regime shifts are more realistic signatures of economic systems. Mathematical tractability, which is undoubtedly a good in itself, should not lead us to overlook this fact. In order to deal with instability-prone phenomena like increasing returns or leverage we may need to design new regulatory mechanisms other than homeostasis-based.

\section{Perfectly rational maximizers}

Selfishness is one of the most reliable characteristics of human beings, but that empirically testable statement does not preclude other forms of economic interaction. Human societies are based on large-scale cooperation among genetically unrelated individuals [9]. Darwin recognized early on that altruistic exchange was problematic for the theory natural selection. Social norms that favor cooperation should have been discounted by natural selection due to the reduced fitness of altruistic individuals. Group selection would endorse communities that contain altruist individuals; this would qualified cooperative groups for an adaptive advantage over groups of selfish individuals. However, groups with individuals prone to self-sacrifice for the common good would attract "free riders" that would join the group to benefit by the rest of the group without paying any of the costs. Furthermore if the free rider character were heritable they would become the fittest transforming an altruistic group into a parasitic one. Biologists have not reach a 
consensus about the legitimacy of group selection. Cultural group selection needs also to be considered, as cooperation in human societies is codified in social norms. Groups that enforce cooperative norms are more likely to prevail ingroup conflicts [10]. Thus, human behavior can not be reduced to individual fitness maximization, they also cooperate in different ways, including reciprocal altruism [11], punishment-based altruism [12], reputation based altruism $[13,14]$, etc.

The egalitarian assumption that all the participants in an economic system are utility maximizers has a practical motivation; it likens economic modeling to an optimal problem. In doing so, no attention is paid to stability because optimal systems are in general stable [15]. From a control theory perspective the Keynesian and the Friedman-Lucas approaches share the idea of the omniscient controller. In a Keynesian's stance, the state is the controller that aims to stabilize the system, and not the mere input as in, for example, deficit spending. On the other hand, the Friedman-Lucas approach relegates the state to a trivial role, that to provide a constant input e.g. money supply, in order to have $\mathrm{n}$ rather controllers than 1. However, in this decentralized approach, there is implicitly a controller - the economist- who defines the objective function: utility maximization, which is typically the wealth, or even better the logarithm of the wealth. Orthodox economic policies have a Panglossian view of economic participants with identical interests competing in the best possible world, which only exists in textbooks of macroeconomic modeling. We need to take a more realistic and honest view: decision makers behavior may largely deviate from optimal criteria, utility functions may not be computable, and people may make systematic errors in predicting the future driving the system to multiple equilibria and instability.

\section{Criticisms to classical economics}

While there is a clear perception at all levels in society that the world economy is in crisis, the economic predicament of the Washington consensus - unregulated global free markets- has not been effectively challenged. The flaws and pitfalls of classical economics succinctly described in the four above-mentioned points have been either amended or criticized from either adherents or critic of the Washington consensus. For example, causality in financial markets has been contested by the business magnate Soros with "reflexivity"-a two-way feedback mechanism in which economic facts e.g. stock prices, shape the agent's thinking and economic facts are affected by the agent's expectations [16]. Alan Greenspan, ex-chairman of the Federal Reserve of the United State, once a believer on the obsolescence of economic cycles in a new era of stable growth driven self regulated free markets, acknowledged later that this was a flawed belief
[17]. However, new classical economics continue to be inescapable in university departments both in teaching and research. Furthermore, global centers of politic and economic power such as the World Bank or the IMF still endorse a body of economic theory that promotes minimal government intervention driven by fiscal responsibility and deficit reduction, mainly by means of spending cuts and consumption tax increases.

The prevalence of the free market ideology, despite the pitfalls in classical economic theory and the wide fluctuations that, for example, financial markets engender, cannot be fully grasped if the political dimension is not taken into account. A global free market is a political project and not any natural outcome embedded in an "unstoppable historical process" such as globalization. For example, the repealing of the Glass-Steagall act of 1932, removed the firewall that policy-makers have imposed in the financial industry that separated commercial and investment banking and successfully prevented the occurrence of instabilities. The GrammLeach-Bliley Act of 1999 was officially passed by the Clinton's administration to help American banks to compete in the global markets of capital, transforming the banking industry into a highly interconnected network extremely vulnerable to the failure of the important hubs in the banking network.

Despite the enormous damage of the financial crisis of 2008 effective reforms in the financial system have been curbed by those with a vested interest in maintaining the status quo. For example, the banking establishment is well aware that the government will guarantee bank liabilities of key credit and insurance institutions whose failure would certainly provoke a systemic contagion in the entire financial system, putting in jeopardy the payment system. Thus, the main bottleneck may not be technical but political $[18,19]$. A strong political will is required to curb the control that "too big to fail banks" hold on the entire economic system. Nonetheless, political action in order to be effective and serve the common good must be informed by up to date scientific studies. For example, in 2009 one major Spanish bank was created -with a broad consensus among the parliamentary political groupings- from the conglomeration of seven small regional saving banks. Some months later the government nationalized the bank. Were those policy makers aware of network-based studies of financial contagion, this could have resulted in a much less harmful outcome for the taxpayer. As it is shown in [20], the creation of conglomerates of banks, lowers diversity across the system as whole, and increases the systemic risk of failure.

The field of futures studies - an interdisciplinary research framework at the intersection of political and social aspects grounded in physical and life science and engineering- may play a protagonist role in proposing new avenues and downto-earth strategies to reunite the dynamism of capitalism 
(creative destruction driven by technological innovations) with societal stability.

\section{A new outlook to economic modeling}

The core tenet of this work is that the most important problems that natural and social science are facing today are inverse problems, and that a new approach that goes beyond optimization, and takes into account the subjective knowledge of the agent is necessary. The inverse problem is ubiquitous in science; molecular structural reconstruction; metabolic network construction; biomedical imaging reconstruction of tumors; reconstruction of the internal structure of the earth; cognitive modeling or option-pricing model in financial markets, are all examples of the inverse problem.

\section{Inverse thinking}

Plato's famous allegory of the cave provides a poetic expression of the inverse problem. The only knowledge that men inside the cave can obtain of real objects, come through shadows cast upon the walls. This is the inverse problem of perception [21]. Inverse Problem theory is extracting information from indirect measurements.

The general problem of learning or to infer the unknown causes from the known effects is an inverse problem which can be studied with either an engineering approach or a purely statistic one. In the former, the causes are the inputs of the system and the effects are the outputs, the deviation or error between the desired output, which is a priori, and the actual output is feedback via the control system, which is dedicated to reduce the error. From a statistical standpoint, input and output data from the training sample that will be used to infer the model parameters that allow us to make accurate predictions of future occurrences. The crucial difference between these two approaches is that in the first case a model of the system is available and in the second the model system is missing and needs to be estimated.

To explain, for example, how an increase in interest rate will cause aggregate demand to decline, we have to study causality. However we cannot trace linear causal chains in complex economic systems such as national economies. Other forms of causality like circular, downward or multiple causality need to be taken into account. We need to foster an "inverse thinking" approach to study causality and related issues e.g. predictability. Predictability can be seen as a forward model that takes a cause $C$ into an effect $E$. The forward problem or modelization allows making predictions on the value of observables, and the inverse problem uses those predictions to infer the values of the parameters that characterize the system. Thus the inverse problem entails the existence of a forward problem to be inverted. To predict the value of an observable, for example a system's output given its input is a forward problem, and to establish a causal link between two observables is an inverse problem. The key aspect of the problem of inference which is an inverse problem, that is, to infer value parameters based on measurements, resides on being able to make explicit any available a priori information about the system being modeled.

Causality is an inverse problem and needs to be studied as such. Thus, very schematically $\operatorname{inv}(f w d(C))=C^{\prime}$ states that if $C=C^{\prime}$ the inverse problem has an unique solution so we have a linear causality, otherwise, $C \neq C^{\prime}$, the inverse problem is illfounded because two causes $C$ and $C^{\prime}$ produce the same effect $E$. Within an inverse thinking approach we don't try to solve an inverse problem that is ill-founded, as for example in $C$ (interest rate increase) causes $E$ (aggregate demand decrease), but we collect all the forward models, that is, all the predictors that are not falsified by data. Thus, we build a set of possible causes or forward model solutions rather than one prediction. The rationale behind this manifold approach to causality is that in order to deal with uncertainty and volatility we need to have a multiple and adaptable hypothesis scheme.

Inverse thinking challenges the orthodox linear causal chain of reasoning, as dramatic system disruptions do not necessarily need large perturbation to occur. The approach is speculative and Popperian in the sense that proposes axioms and predictions that can be refuted. The inverse thinking approach will help to discover the design principles for robust systems in the face of uncertainty and complexity $[22,23]$.

\section{Bio-inspired homeostasis}

Conceptual clarification and epistemic discipline in the misuse of analogies between physics and economy is a requisite for developing comprehensible economic knowledge, independently of the particular domain in which it is used. Economies are not mere exchange systems, the transformation of labor and energy into tangible goods or production is also part of real economic systems. From a thermodynamic point of view, the term economic equilibrium is an oxymoron. Equilibrium is a frozen state of no change, where no macroscopic flows exist. Thus, it is a concept more likely to apply to closed or isolated systems rather than to real economies. Among other things, financial crashes have very acutely showed that the conception of economic equilibrium based on the mechanical analogy of a pendulum is untenable. The benefit attained in terms of mathematical tractability by adopting the hypothesis that economic agents achieve equilibrium by maximizing utility, must not lead us to neglect basic facts. Economic agents, value goods and services in order to take decisions referred to those economic assets in an attempt to forecast a favorable outcome. But they decide 
so in multiple ways according to ecological and historical contexts. Moreover, their actions have one direction that goes from the irreversible past to the uncertain future. Thus, static equilibrium methods are not applicable to real economies when these aspects are considered.

Non-equilibrium statistical mechanics may provide a more fruitful analogy than Gibbs' theory of equilibrium thermodynamics [24]. However the concept of non-equilibrium steady state needs to be carefully addressed as a process that needs to be maintained by the interplay of non-zero flows of energy or matter, rather than a final state as equilibrium. Future research should try to develop scenarios with $n$ different equilibria with different dynamic regimes. The inverse thinking approach may provide the necessary mindset to strengthen our understanding of multiple equilibria, as it natively deals with a scenario of multiple and instable solutions, including "bubble solutions" or large deviations from equilibrium. To turn these theoretical insights into real applications, we need to develop biologically inspired models of stability mechanisms, in particular homeostasis [25]. A new view of homeostasis that deals with integrated outcomes rather than single signals is already being promoted in biomedical applications [26]. The notion of stability as a homeostatic behaviour confined within a static set of admissible values is here challenged. For example, nervous systems effectively solve an important conundrum: allow synaptic plasticity without compromising the stability and integrity of the system. Hebbian learning [27] which is considered the cornerstone of neural learning is problematic because synapses that are better than others at activating a neuron, will be strengthen in a positive feedback loop that reinforce synapsis that are correlated with neural firing and penalize those that are not. A number of homeostatic plasticity models have been suggested to compensate for the inherently unstable dynamics that is at the basis of learning-induced changes in synaptic strength [28]. More recently, the concept of network homeostasis - the coordinated action of individual regulators in a network- is being developed [29]. In this view, homeostasis is a highly dynamic process accomplished by means of coordinated adjustments in network interconnectivity.

From a futures research perspective, the analogy between Hebbian learning rules and unregulated capitalism, both inherently unstable as "the rich get richer", need to be seriously considered. Economics may be called to act as a natural bridge able to connect social and technological aspects. This positioning may sound extremely risky, the recent financial meltdown and the inability of the economic models to forecast these extreme events, has done nothing but reinforced the old motto "economics is the dismal science".

\section{Utility}

Non-parametric estimators can be arbitrarily well approximated, this property is called consistency and it is the major reason for the popularity of model-free estimators. Consistency guarantees that, for a sufficiently big training data set, it is possible to achieve an optimal performance for any inference-learning task. Thus, there is apparently a causal connection between consistency and optimality. However, non-parametric estimators are optimal because they are consistent, but consistency is an asymptotic property. In real problems, the training data set cannot be assumed to be arbitrarily big -data samples may be small or have dispersed distribution. This point has direct consequences in economic policy. The standard approach in optimal economic policy, which is mainly concerned with performing a gradient ascent on utility or wealth maximization, should be critically revisited. Modeling all economic systems as optimal utility maximizers is at least questionable using empirical standards, as it depends on consistency, which is an asymptotic property. The Allais' paradox [30] can be seen as a reflection of the empirical limitation of using optimal methods when consistency may not necessarily apply. Indeed the same person that prefers an apple to an orange, may prefer one dollar and one orange to one dollar and one apple. The idea of using an optimization algorithm to forecast people's choice as represented in the utility function is untenable [31]. We need to transcend the idea of one consistent and optimal criterion for all the participants behavior, proposing a multifold space of subjective utility models, represented by a $n$ dimensional set of subjective expected utility (SEU). In order to take into account how individual SEU maximization affects and is affected by the aggregate SEU, each SEU is considered within an ecological perspective. The subjective expectations utility function (SEU) needs to be defined in terms of the subjective utility $\mathrm{u}(\mathrm{x})$ and the subjective probability $\mathrm{P}(\mathrm{x})$ for a given action $\mathrm{x}$. Thus, the view that is adopted here goes beyond modeling economic agents as profit optimizers, to focus on the aggregate level, by studying the dynamics of economic networks of large numbers of agents equipped with subjective beliefs operating under conditions of uncertainty. It ought to be remarked that SEU function is a non-convex utility function, so it is not the same as Bayesian utility maximization.

Economies emerge out of subjective beliefs of agents, endowed with internalized models that try to adapt to the aggregate patterns that their actions and expectations create. The SEU models the looking forward capacity of economic cognitive agents. By including the cognitive and ecological dimensions that are the basis of the agent's behaviour, we will allow endogeneity in the structure of the inverse problem and will be in a better disposition to answer questions regarding which topological architecture is more prone to systemic failure and gain insight for the design principles to build more resilient networks.

The inverse problem can be effectively tackled by introducing all the available information or bias to constrain the 
model space of predictors represented by subjective utility functions. Bias or a priori knowledge, works as a selection mechanism over the space of falsifiable models, which in turn are tested against the data, that is, those that do not predict data within an established criterion are discarded or falsified in Popperian parlance. Thus, it is within the inverse thinking perspective that we can start understanding the computational goal(s) the economic systems under study.

\section{Discussion}

Our zeitgeist is that of deregulation and credit. The political links of special interest groups were not the only driving force that pushed economies in this state of affairs. At a more fundamental level, deregulation can be seen as an anticipated outcome of the increase in complexity in the world network economy that globalization has brought about. In the 70's stabilization policies designed to for example control in inflation and unemployment using optimal control theory, did not succeed [32, 33]. National economies became too complex to be regulated with that toolset. Monetarist economists' credo that the system itself could self-regulate without any need to use an external controller was championed by policy makers. The view that complexity was beneficial for its stability was widely assumed in ecology studies, until the 70s when the mathematical biologist Robert May demonstrated that complexity might engender instability [34]. Apparently, economists were unaware of this work, as the standard economic modeling of optimal forecasters in stable equilibrium remained relatively undisputed until the 2008 financial crisis. Mainstream economic theory still relies upon Newtonian mechanics and axioms and assumptions with not empirical justification. Whether this is just an anachronism or a mathematically-based ideology [35] constitutes a rich discussion forum for the field of futures studies.

By focusing in the inverse problem we may reduce the isolation of economic theories from the natural science, as it provides an ideal platform where related problems from different disciplines can be studied under a common approach with comparable results. The foundations of modern economic theory e.g., efficient market hypothesis, rational choice theory fueled by the "physics envy" of neo classical economists has shown itself to be fatally flawed. We sorely need to develop a new theoretical framework, based on realistic scenarios in which the plurality of internal motivations of the economic agents (individuals, firms, institutions), help us to establish a systemic understanding of complex socio-economic systems.

New means to study quantities that are not directly observable like utility, drawing causal maps with measurements gathered from financial economics, need to be scrutinized. By adopting an inverse perspective, we contribute to the creation of new ways of economic thinking and to the discovery of new tools to tackle inverse problems. In doing so, the economic discipline will be emancipated from its ties to XIX century physics to enter into a new paradigm shift. A new picture of stability will emerge from the study of the cognitive and ecological dimensions in economic systems, grounded in the conceptual and methodological apparatus provided by the inverse problem theory. This paper intends to be a commencing contribution towards a new foundation for complex economic systems modeling, sustained by a plurality of future objectives to be undertaken in a multidisciplinary and mathematically innovative way.

Open Access This article is distributed under the terms of the Creative Commons Attribution License which permits any use, distribution, and reproduction in any medium, provided the original author(s) and the source are credited.

\section{References}

1. Akerlof GA (2002) Behavioral macroeconomics and macroeconomic behavior. Am Econ Rev 92(3):411-433

2. Glimcher PW (2003) Decisions, uncertainty, and the brain: The science of neuroeconomics. A Bradford Book, Cambridge

3. Arthur B (1999) Complexity and the economy. Science 284(5411):107-109

4. Friedman M (1966) Essays in positive economics. University of Chicago Press, Chicago

5. Wigner E (1960) The unreasonable effectiveness of mathematics in the natural sciences. Commun Pur Appl Math 13(1):1-14

6. Schumpeter JA (1994) Capitalism, socialism and democracy. Routledge, London

7. Lucas R (1976) Econometric policy evaluation: A critique. In: Brunner K, Meltzer AH (eds) The Phillips curve and labor markets. Carnegie-Rochester Conference Series on Public Policy, NorthHolland, pp 19-46

8. Cowan R, Rizzo M (1996) The genetic causal tradition of modern economic history. Kyklos 49(3):273-317

9. Fehr E, Fischbacher U (2004) Social norms and human cooperation. Trends Cognit Sci 8(4):185-190

10. Rosenberg A (2008) Philosophy of biology a contemporary introduction. Routledge, New York

11. Stevens JR, Hauser MD (2004) Why be nice? psychological constraints on the evolution of cooperation. Trends Cognit Sci 8(2):60-65

12. Panchanathan K, Boyd R (2004) Indirect reciprocity can stabilize cooperation without the second-order free rider problem. Nature 432(7016):499-502

13. Gintis H, Smith EA, Bowles S (2001) Costly signaling and cooperation. J Theor Biol 213(1):103-119

14. Milinski M, Semmann D, Krambeck H-J (2002) Reputation helps solve the tragedy of the commons. Nature 415(6870):424-426

15. Astrom KJ (2006) Introduction to stochastic control theory. Dover Publications, New York

16. Soros G (2008) The alchemy of finance. Wiley, Hoboken

17. Fleckenstein WA, Sheehan F (2008) Greenspan's bubbles: The age of ignorance at the Federal Reserve. McGraw-Hill, New York

18. Sornette D, von der Becke S (2011) Complexity clouds financerisk models. Nature 471(7337):166 
19. Admati A, Hellwig M (2013) The bankers' new clothes: What's wrong with banking and what to do about it. Princeton University Press, Princeton

20. Haldane AG, May RM (2011) Systemic risk in banking ecosystems. Nature 469(7330):351-355

21. Groetsch CW (1993) Inverse problems in the mathematical sciences. Vieweg, Hamburg

22. Tarantola A (2006) Popper, Bayes and the inverse problem. Nat Phys 2:482-484

23. Gomez-Ramirez J, Sanz R (2013) On the limitations of standard statistical modeling in biological systems: a full bayesian approach for biology. Progr Biophys Mol Biol. doi:10.1016/j.pbiomolbio.2013.03.008

24. Bergersen B (2009) Statistical mechanics of utility and equilibrium: analogies between economics and statistical physics. Phys Can 65(4):205-209

25. Simon HA (2002) Near decomposability and the speed of evolution. Ind Corp Chang 11(3):587-599

26. Hotamisligil GS (2006) Inflammation and metabolic disorders. Nature 444(7121):860-867

27. Hebb DO (1949) The organization of behavior: A neuropsychological theory. John Wiley and Sons, Inc., New York, p 335
28. Turrigiano GG, Nelson SB (2004) Homeostatic plasticity in the developing nervous system. Nat Rev Neurosci 5(2):97-107

29. Maffei A, Fontanini A (2009) Network homeostasis: a matter of coordination. Current Opinion in Neurobiology 19(2):168-173

30. Quiggin J (1993) Generalized expected utility theory: The rank-dependent expected utility model. Kluwer-Nijhoff, Amsterdam

31. Kurz M (2008) Beauty contests under private information and diverse beliefs: how different? J Math Econ 44:762-784

32. Prescott EC (1977) Should control theory be used for economic stabilization? In: Brunner K, Meltzer AH (eds) Carnegie-rochester conference series on public policy, 7th edn. North-Holland, Amsterdam, pp 13-38

33. Kalchbrenner JH, Tinsley PA (1977) On filtering auxiliary information in short-run monetary policy. In: Brunner K, Meltzer AH (eds) Carnegie-rochester conference series on public policy, 7th edn. North-Holland, Amsterdam, pp 39-84

34. May R (1972) Will a large complex system be stable? Nature 238:413-414

35. McCauley JL (2000) The futility of utility: how market dynamics marginalize Adam Smith. Phys Stat Mech Appl 285(3-4):506-538 